\title{
Choosing and estimating shear resistance parameters of gravel soils
}

\author{
Anatoly Mirnyy ${ }^{1, *}$, Valeriy Merkin ${ }^{1}$ \\ ${ }^{1}$ Moscow State University of Civil Engineering, 129110, Yaroslavskoe shosse, 26, Moscow, Russia
}

\begin{abstract}
This issue is dealing with new yield criterion for gravel soils development. The most popular criteria for non-cohesive soils are analysed and the parameters they are based on. Proposed yield criterion is based on invariant parameters and concerns friction, cohesion and engagement between particles. It also takes into account second principal stress by using a non-fixed sliding plane. The parameters of this criteria are physically justified and can be determined by standard soil test.
\end{abstract}

\section{Introduction}

Under the soil strength its shear resistance is usually recognized. It is a fundamental soil parameter, and it's appropriate esteem is vital for rational design of numerous constructions, especially soil dams. Shear resistance of soil and its strength is determined by: a) inner friction forces, depending on normal stress; b) reversible cohesion of watercolloid nature, c) rigid structural cohesion [1,14-17]. Structural cohesion is normal for rock soils and usually is not considered in disperse soils. Thus, the most rational method for fine-grained soils shear resistance description is the use of two-parametrical yield criterion.

One of the most well-known yield criterion is a Mohr-Coulomb criterion [2]:

$$
\sin \varphi=\frac{\sigma_{1}-\sigma_{3}}{\sigma_{1}+\sigma_{3}+2 c \cdot \operatorname{ctg} \varphi}
$$

The Mohr-Coulomb theory considers the sliding plane that intersects the plane of the maximum and minimum principal stresses and is parallel to the axis of the intermediate principal stress. Many authors have noted that the Mohr-Coulomb theory is most suitable for solving problems of a flat stress-strain state, slopes stability problems mostly $[3,4]$.

A significant drawback of this platform is its non-physical hypotesis - intermediate principal stress takes an infinitely large value, its effect is not considered [5]. This leads to an underestimation of the strength, as this plane is the most dangerous, but is no likely to appear in complicated stress-strain state therefore its application to strength and stability calculations of the foundations of most buildings is not recommended.

Experimental studies of different soil in a wide range of stresses $\sigma_{3}$ (from 0 to 4,0 $\mathrm{MPa}$ ), performed by various researcehrs [6-8] have revealed, that the intermediate principal

* Corresponding author: reg@oconnor.ru 
stress has an influence on the shear resistance, hence the yield condition needs to account for this effect.

There was an interesting yield condition (2) performed by A. I. Botkin [5,9], who suggested that the Mohr-Coulomb condition is satisfied on the octahedral plane, equally tilted to the axes of principal stresses:

$\tau_{i}^{*}=\sigma \cdot \operatorname{tg} \varphi_{\text {oct }}+c_{\text {oct }}$.
where $\tau_{i}^{*}=\frac{\sqrt{\left(\sigma_{1}-\sigma_{3}\right)^{2}+\left(\sigma_{2}-\sigma_{3}\right)^{2}+\left(\sigma_{3}-\sigma_{1}\right)^{2}}}{3}$ - shearing stress on the octahedral plane; $\sigma=\frac{\sigma_{1}+\sigma_{2}+\sigma_{3}}{3}$ - normal stress on the octahedral plane; $\varphi_{\text {oct }}$ - inner friction angle for this plane; $c_{o c t} \cdot-$ cohesion for this plane.

This theory takes into account the intermediate principal stress, however, also has some drawbacks. The magnitude of the normal stress on this plane is equal to the arithmetic mean of the three principal stresses. This decision leads to an overestimation of the normal stress. The shearing stress on this site has a small value, as calculated on the basis of deviations of the values of the principal stresses from the average, and obviously cannot be very large.

Malyshev M. V. [5] noted the "phenomenological" character of this theory, as plane selection is dictated by mathematical simplicity. An important element of this theory is the notion of the angle $\theta$, defining the direction of shearing stress acting on the octahedral plane.

The results of the studies summarized by Rasskazov L. N. [10, 11] confirm a significant influence of intermediate principal stress $\sigma_{2}$ on the shearing resistance (up to $10^{\circ}$ ); the nonlinearity of the envelope of the Mohr circles in the majority of cases (reduction of up to $15^{\circ}$ ); the effect on the strength of the loading trajectory and Nadai-Lode parameter (change in the range of $2-4^{\circ}$ ). In addition, to date there is no possibility of accounting for engagement of particles in coarse-grained soils and the resulting increase in strength.

Especially important these phenomena are in the calculation of foundations and earth structures composed of coarse-grained soils with sand or clay filler. At the suggestion of $P$. I. Gordienko [12] to assess the strength of coarse-grained soil using not the $\varphi$ and $c$ parameters, but the shearing angle $\psi$, determined with $c=0$. Each value of $\psi$ has appropriate $\sigma_{1}, \sigma_{n}$ and $\sigma_{3}$ values. Changing of $\psi$ for gravel materials, depending on the stresses at the suggestion of P. I. Gordienko, can be approximately estimated by the formula (3).

$$
\psi_{\sigma}=\psi_{0}-5 \lg \frac{\sigma_{n}}{\sigma_{0}}
$$

Where $\psi_{0}$ - shearing angle at $\sigma_{3} \rightarrow 0, \sigma_{n}=\frac{2 \sigma_{1} \sigma_{3}}{\sigma_{1}+\sigma_{3}}$ - normal stress on the sliding plane; $\sigma_{0}=0,2 M P a$.

Unfortunately, these relationships are markedly empirical and must be adjusted for each site.

At present, a large number of yield and strength conditions where developed for non cohesive soils. Some of them, such as the Matsuoka-Nakai and Lade-Duncan criteria [13], take into account the magnitude of the intermediate principal stress and is applicable at different values of Nadai-Lode parameter, but are single-parameteric. Others, such as the Drucker-Prager criterion, use the same parameters as the Coulomb-Mohr, but have disadvantages, expressed in incorrect results for volume strain and dilatancy. 
In this study the authors made an attempt to develop the yield criterion, taking into account all the described above regularities and phenomena.

The yield condition suitable for the description of shear resistance of coarse soils should meet the following requirements:

- it should consider the influence of intermediate principal stress and type of stress state. This is achieved by consideration of the sliding plane, which is not fixed in space of principal stresses and is determined by the current values of stresses;

- take into account the physical nature of the strength of fine-grained soils;

- parameters for this condition should be invariant, i.e., should not depend on the method of their estimation.

The authors deliberately omitted the question of the shear resistance dependence of soil density, since, in practice, coarse-grained soils are often used when the density is close to maximum, and the compaction during loading is negligible.

The task of developing the yield condition can be divided into three fundamental stages. The first step is to define the most dangerous plane, where all the stresses are considered. The second step is to choose invariant parameters, corresponding to the physical meaning of the described phenomena. Finally, in the third stage it is necessary to establish the mathematical relationship between these parameters and the components of stresses at the selected plane, allowing to describe the nature of the envelope of the Mohr circles observed in the experiment. This division greatly simplifies the solution to this problem, since the definition of the stress state and the identification of plane is a mathematical abstraction, but a yield condition, on the contrary, should have reference to the nature of the phenomenon in the medium.

\section{Choosing a sliding plane}

The account of all three principal stresses is possible when considering the plane, which is determined by the magnitude of the principal stresses at the ultimate state. It is obvious that such a plane will have a different angle relative to each of axes of principal stresses. General view of such a plane is shown in figure 1.

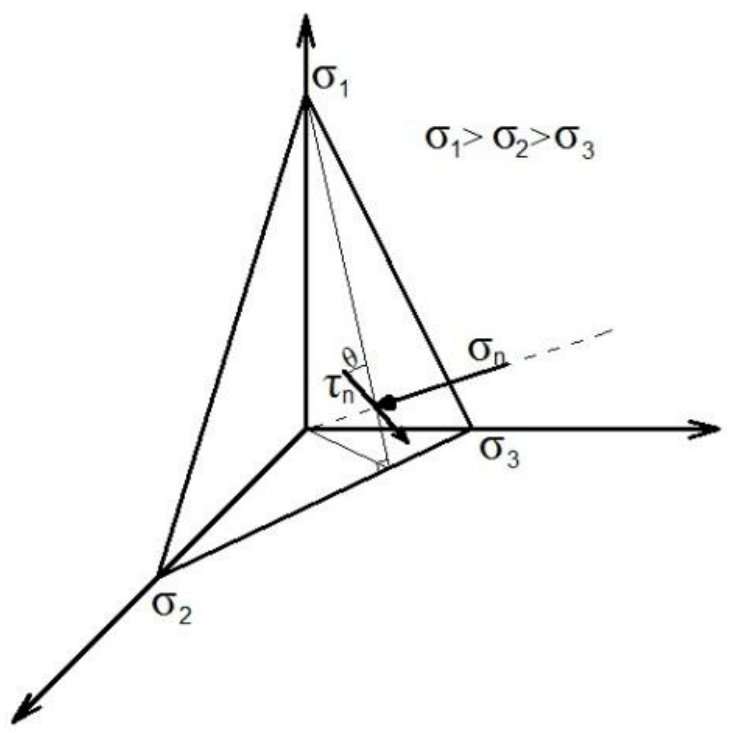

Fig. 1. General view of a suggested plane

The position of the plane may be set by the guiding cosines of the normal vector. The module of this vector is expressed as follows (4): 


$$
l=\frac{\sigma_{1} \sigma_{2} \sigma_{3}}{\sqrt{\sigma_{1}^{2} \sigma_{2}^{2}+\sigma_{2}^{2} \sigma_{3}^{2}+\sigma_{3}^{2} \sigma_{1}^{2}}}
$$

From the consideration of triangles values of the guiding cosines can be obtained:

$$
\begin{aligned}
& \cos \alpha=\frac{l}{\sigma_{1}}=\frac{\sigma_{2} \sigma_{3}}{\sqrt{\sigma_{1}^{2} \sigma_{2}^{2}+\sigma_{2}^{2} \sigma_{3}^{2}+\sigma_{3}^{2} \sigma_{1}^{2}}} \\
& \cos \alpha=\frac{l}{\sigma_{2}}=\frac{\sigma_{1} \sigma_{3}}{\sqrt{\sigma_{1}^{2} \sigma_{2}^{2}+\sigma_{2}^{2} \sigma_{3}^{2}+\sigma_{3}^{2} \sigma_{1}^{2}}} \\
& \cos \alpha=\frac{l}{\sigma_{3}}=\frac{\sigma_{1} \sigma_{2}}{\sqrt{\sigma_{1}^{2} \sigma_{2}^{2}+\sigma_{2}^{2} \sigma_{3}^{2}+\sigma_{3}^{2} \sigma_{1}^{2}}}
\end{aligned}
$$

Using the known formulas of solid mechanics to determine the normal and shearing stresses on any plane we can get the expressions for these stresses:

$$
\begin{aligned}
& \sigma_{n}=\sigma_{1} \cos ^{2} \alpha+\sigma_{2} \cos ^{2} \beta+\sigma_{3} \cos ^{2} \gamma \\
& \tau_{n}=\sqrt{\sigma_{1}^{2} \cos ^{2} \alpha+\sigma_{2}^{2} \cos ^{2} \beta+\sigma_{2}^{3} \cos ^{2} \gamma-\sigma_{n}^{2}}
\end{aligned}
$$

After substituting the previously obtained values of the guiding cosines (5-7) these expressions take the form:

$$
\begin{gathered}
\sigma_{n}=\frac{\left(\sigma_{1} \sigma_{2} \sigma_{3}\right)\left(\sigma_{1} \sigma_{2}+\sigma_{2} \sigma_{3}+\sigma_{3} \sigma_{1}\right)}{\sigma_{1}^{2} \sigma_{2}^{2}+\sigma_{2}^{2} \sigma_{3}^{2}+\sigma_{3}^{2} \sigma_{1}^{2}} \\
\tau_{n}=\frac{\sigma_{1} \sigma_{2} \sigma_{3}}{\sigma_{1}^{2} \sigma_{2}^{2}+\sigma_{2}^{2} \sigma_{3}^{2}+\sigma_{3}^{2} \sigma_{1}^{2}} \sqrt{3\left(\sigma_{1}^{2} \sigma_{2}^{2}+\sigma_{2}^{2} \sigma_{3}^{2}+\sigma_{3}^{2} \sigma_{1}^{2}\right)-\left(\sigma_{1} \sigma_{2}+\sigma_{2} \sigma_{3}+\sigma_{3} \sigma_{1}\right)^{2}}
\end{gathered}
$$

These values of normal and shear stresses are proposed to use in determining invariant sets of the strength parameters.

\section{Choosing the invariant strength parameters}

Many years of experience in the application of the Mohr-Coulomb criterion in soil mechanics has shown that the two-parameter model is a reasonable representation of the shear strength of fine-grained soils. However, studies on the shear resistance of coarsegrained soils in a wide range of stresses show poor convergence with experimental observations. In the works of many researchers, in particular, Boldyrev, G. G., the discrepancy in the values of the strength parameters obtained by various methods is shown. This fact leads to the conclusion about insufficiency of our ideas about the nature of strength of fine-grained soils.

First and foremost this statement refers to evaluation of internal friction through internal friction angle. Internal friction in dispersed soils is caused not only by sliding friction the Amontons-Coulomb, but the rolling friction when turning the particles, the roughness and roundness of the particles and, consequently, their engagement. Obviously, part of these phenomena does not depend on the normal stress applied and can be related to the cohesion forces.

At the same time, the strength parameters are a function of the loading rate. For example, during very slow loading, internal links partially recover and sliding friction is not transformed into rolling friction.

Thus, as the invariant of the strength parameters of the soil the following should be considered: 
- inner friction parameter $\varphi_{i}$, for sliding friction determined by soil mineralogy. If $\varphi_{i}=f(\dot{\gamma})$ is considered peak strength could be accounted for. As a residual value of $\varphi_{i}$ the natural slope angle should be taken;

- cohesion parameter $c$, reflecting the influence of internal relations, regardless of their reversibility and applied stresses. It is constant, due to physical and chemical parameters of the soil;

- engagement parameter, reflectiong particle roughness and its influence on shearing resistance.

The most difficult task is to identify the role of engagement in the shear strength of finegrained soils. From physical sense of engagement, it follows that its magnitude does not depend on the compression, as even in the absence of compression with only shear forces applied there is a resistance due to engagement. The magnitude of this resistance will be constant for an infinitely strong material, as the destruction of the engagement irregularities will not occur. With decreasing strength of the material the irregularities will begin to split. In the course of the shearing theret will be gradual bridging of the bumps. Finally in the case of extremely fragile material, engagement will not be observed, since the shear plane will pass through the material of the particles. This mechanism is presented in figure 2 .

A

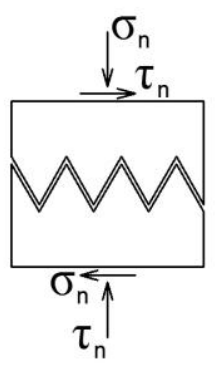

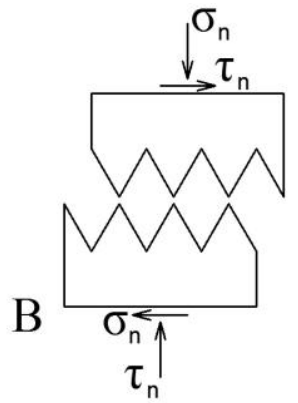
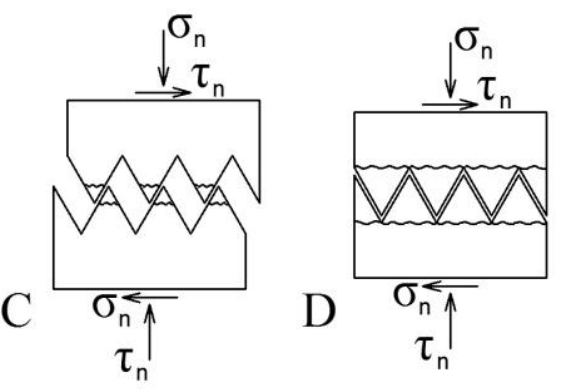

Fig. 2. A) - starting position; B) - infinite strength; C) - finite strength; D) - no shearing resistance.

At the same time, the magnitude of compression will change the amount of friction, the magnitude of the normal force to the surface of the prong is greater than the magnitude of the force normal to the cut surface due to the tilt of prongs. Chipping of prongs will occur when the shear stress exceeds the shear strength of the particles. Thus, the contribution of the engagement in shear resistance is easier to evaluate in the form of increased friction. Engagement is implemented before the start of the shearing prongs, and then begins to decline to zero with smoothing irregularities.

\section{The invariant yield criterion development}

Yield criterion is taking the form:

$$
\tau^{*}=\sigma \cdot \operatorname{tg} \varphi \cdot\left(1-(1+n)^{\sigma-R}\right)+c
$$

where $\mathrm{R}$ - axial compression resistance of particles material,

$n$-parameter, depending on shear resistance of particles material.

This yield criterion takes into account the shear resistance due to the engagement and the gradual decrease due to the prongs shearing.

The parameter $\mathrm{n}$ can be expressed, provided that we know the shear resistance of the particles, however, it is more convenient to define it on the basis of experimental data. It should be guided by the following values:

When $n=0$ - there is no friction in shear resistance; 
When $n=0,2$ the prongs are nearly indestructable.

On the figure 3 there is a graph of a limit line function $\tau^{*}=f(\sigma)$ with different $n$ values.

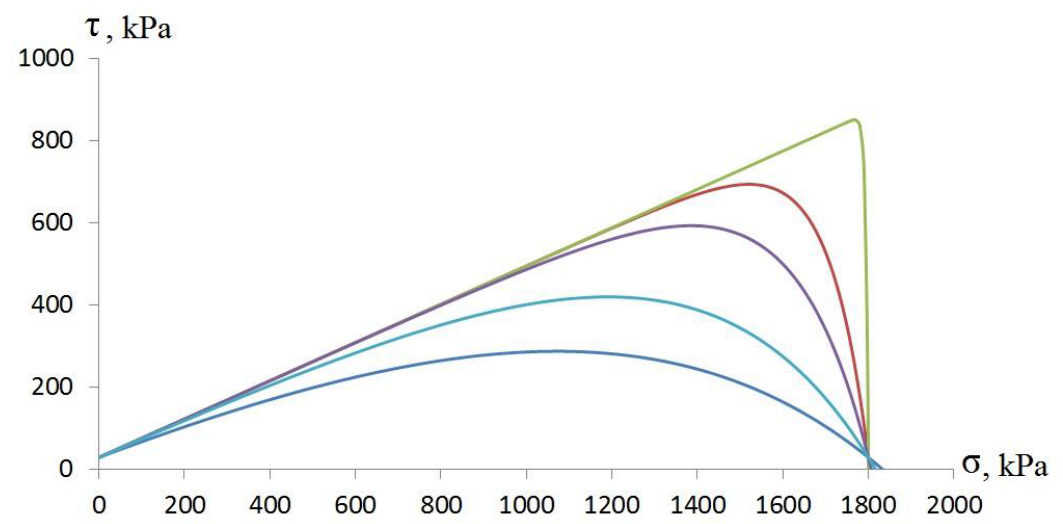

Fig. 3. Limit lines with different values of $n$.

It is obvious that such a view explains the definition of engagement by many authors as an additional constant component close to cohesion. This is due to the application of the linear form of the limit line. Holding such line through any three points of the curve shows initial shear strength, different from zero, which is interpreted as "engagement".

In its final form, the proposed yield criterion can be formulated in the form of three equations, allowing to determine the stress components for the sliding plane and the interaction between them:

$$
\left\{\begin{array}{c}
\sigma_{n}=\frac{\left(\sigma_{1} \sigma_{2} \sigma_{3}\right)\left(\sigma_{1} \sigma_{2}+\sigma_{2} \sigma_{3}+\sigma_{3} \sigma_{1}\right)}{\sigma_{1}^{2} \sigma_{2}^{2}+\sigma_{2}^{2} \sigma_{3}^{2}+\sigma_{3}^{2} \sigma_{1}^{2}} \\
\tau_{n}=\frac{\sigma_{1} \sigma_{2} \sigma_{3}}{\sigma_{1}^{2} \sigma_{2}^{2}+\sigma_{2}^{2} \sigma_{3}^{2}+\sigma_{3}^{2} \sigma_{1}^{2}} \sqrt{3\left(\sigma_{1}^{2} \sigma_{2}^{2}+\sigma_{2}^{2} \sigma_{3}^{2}+\sigma_{3}^{2} \sigma_{1}^{2}\right)-\left(\sigma_{1} \sigma_{2}+\sigma_{2} \sigma_{3}+\sigma_{3} \sigma_{1}\right)^{2}} \\
\tau^{*}=\sigma \cdot \operatorname{tg} \varphi \cdot\left(1-(1+n)^{\sigma-R}\right)+c
\end{array}\right.
$$

It should be noted that the friction angle in this criterion is not numerically equal to the friction angle for other yield conditions, in particular the Mohr-Coulomb and von MisesSchleicher-Botkin.

The technique of definition of parameters in general is similar to conventional test methods, however, when interpreting data it is necessary to consider the range of stresses in the test.

The angle of internal friction and cohesion should be determined according to the initial part of a limit line, where even in the area of stress concentration the strength of the particles is not exceeded.

\section{Conclusions}

1. The most common yield criteria have limited application for coarse-grained soils, as engagement and the decrease in shear resistance with increasing normal stress is not fully taken into account.

2. A yield condition suitable for coarse-grained soils must consider the engagement of the particles and enable the strength of the particles as a parameter 
3. The proposed yield condition can be used to describe the limit line, taking into account its nonlinearity.

4. The parameters of is yield criterion can be determined on the basis of experimental data.

\section{Acknowledgements}

This work was financially supported by the Ministry of Russian Education (state task $\# 7.1576 .2014 / \mathrm{K})$

\section{References}

1. N.N. Maslov, Soil mechanics and engineering geology basics (Vysshaya Shkola, Moscow, 1968)

2. Z.G. Ter-Martirosyan, Soil mechanics (ASV, Moscow, 2009)

3. J. M. Duncan, S.G. Wright, Soil Strength and Slope Stability (John Wiley \& Sons, Inc., USA, 2005)

4. G. K. Clein, Structural mechanics of granular medium (Stroizdat, Moscow, 1977)

5. M.V. Malyshev, Soil strength and stability of foundations of structures (Stroizdat, Moscow, 1994)

6. S.M. Ataya, Gravel soils shear resistance studies in triaxial machine (VODGEO, Moscow, 1965)

7. T. Mendoza, The strength of coarse-grained soils with sand filler in terms of spatial stress state (MISI, 1984)

8. A.L. Kryzhanovskiy, T. Mendoza, E. Ukibaev, Eng. Geol. 2, (1985)

9. G.G. Boldyrev, Soils mechanical properties obtaining methods (PGUAS, Penza, 2008)

10. A.L. Goldin, L.N. Rasskazov, Soil dams design (ASV, Moscow, 2001)

11. L.N. Rasskazov, Gravel soils shear resistance (VODGEO, Moscow, 1968)

12. P.I. Gordienko, Some design issues of high stone and soil dams (MISI, 1960)

13. L. Mengcheng, C. Yufeng, L. Hanlong, Int. Jour. of Rock Mech. and Mining Sc. 50 (2012)

14. A.Z. Ter-Martirosyan, Z.G. Ter-Martirosyan, V.V. Sidorov, Soil Mechanics and Foundation Engineering 1, 1-8 (2016)

15.Z.G. Ter-Martirosyan, E.S. Sobolev, A.Z. Ter-Martirosyan, International Journal of Applied Engineering Research 11(3), 1719-1723 (2016)

16. A.Z. Ter-Martirosyan, Z.G. Ter-Martirosyan, Procedia Engineering 111, 756-762 (2015)

17.Z.G. Ter-Martirosyan, A.Z. Ter-Martirosyan, Soil Mechanics and Foundation Engineering 51 (6), 273-281 (2015) 